\title{
UNIVERSAL FILTRATION OF SCHUR COMPLEXES
}

\author{
GIANDOMENICO BOFFI
}

(Communicated by Eric Friedlander)

\begin{abstract}
The Schur complex $\mathbf{L}_{\lambda / \mu} \phi$ has proved useful in studying resolutions of determinantal ideals, both in characteristic zero and in a characteristic-free setting. We show here that in every characteristic, $\mathbf{L}_{\lambda / \mu} \phi$ is isomorphic, up to a filtration, to a sum of Schur complexes $\sum_{\nu} \gamma(\lambda / \mu ; \nu) \mathbf{L}_{\nu} \phi$, where $\gamma(\lambda / \mu ; \nu)$ is the usual Littlewood-Richardson coefficient. This generalizes a well-known direct sum decomposition of $\mathbf{L}_{\lambda / \mu} \phi$ in characteristic zero.
\end{abstract}

\section{INTRODUCTION}

Let $F$ and $G$ be finitely generated free $R$-modules of ranks $m$ and $n$, respectively, and $\varphi: G \rightarrow F$ be an $R$-module homomorphism. To every skew shape $\lambda / \mu$, associate the Schur complex of $\varphi$ of shape $\lambda / \mu$, as defined in [2] and denoted by $\mathbf{L}_{\lambda / \mu} \varphi$. It is well known that if $R$ contains a copy of the rationals, then $\mathbf{L}_{\lambda / \mu} \varphi$ is isomorphic to $\sum_{\nu} \gamma(\lambda / \mu ; \nu) \mathbf{L}_{\nu} \varphi$, where $\mathbf{L}_{\nu} \varphi$ is the Schur complex associated to the shape $\nu$ and $\gamma(\lambda / \mu ; \nu)$ is the ordinary Littlewood-Richardson coefficient. In this paper, we construct a universal (= independent of the ring $R$ ) filtration of $\mathbf{L}_{\lambda / \mu} \varphi$, whose associated graded object precisely coincides with $\sum_{\nu} \gamma(\lambda / \mu ; \nu) \mathbf{L}_{\nu} \varphi$.

One should remark that the above can be applied to all complexes $\mathbf{L}_{\lambda_{1} / \mu_{1}} \varphi \otimes$ $\mathbf{L}_{\lambda_{2} / \mu_{2}} \varphi \otimes \cdots \otimes \mathbf{L}_{\lambda_{t} / \mu_{t}} \varphi$, which are further instances of Schur complexes associated to skew shapes.

The techniques used in the construction are those introduced in the author's thesis, Brandeis University, 1984 (largely reproduced in [4]), and successfully exploited in $[5,3,6]$. In fact, all the results contained in these four papers can be obtained as corollaries of the result for $\mathbf{L}_{\lambda / \mu} \varphi$.

One expects the universal filtration of $\mathrm{L}_{\lambda / \mu} \varphi$ to assist in solving some problems akin to those which originated the notion of Schur complex (cf. $[1,7$, 9], etc.). For instance, such a filtration is an ingredient for the study of the homology of the complex introduced in [11].

It has to be pointed out that in the very special cases when $\mu=(t)$ and $\mu=\left(1^{t}\right)$, a universal filtration for $\mathbf{L}_{\lambda / \mu} \varphi$ has been already described in [8] but in a way which is of little help to us here. (In fact, in his thesis, written before

Received by the editors February 10, 1992.

1991 Mathematics Subject Classification. Primary 13D25; Secondary 14M12, 15 A72.

The author was partially supported by M.U.R.S.T. and is a member of C.N.R.-G.N.S.A.G.A. 
[8], Ko believed to have the description of a universal filtration for every Schur complex, but there were some errors.)

Notation and basic facts are freely borrowed from [2].

\section{Preliminaries}

We closely follow the strategy outlined in $[5, \S 2]$.

We first define an auxiliary free $R$-module $H$; namely, we set $H=R^{s}$, where $s=\mu_{1}$ (the length of the first row of $\mu$ ). We then call $\psi$ the composite morphism $G \stackrel{\varphi}{\rightarrow} F \stackrel{\text { inc }}{\rightarrow} F \oplus H$.

Choose ordered bases $Y=\left\{y_{1}, \ldots, y_{m}\right\}$ and $X=\left\{x_{1}, \ldots, x_{n}\right\}$ for $G$ and $F$, respectively (both of them ordered according to the subscripts), and denote by $Z=\left\{z_{1}, \ldots, z_{s}\right\}$ the canonical basis of $H$. Let $S=Y \cup X \cup Z$. Thinking of $S$ as ordered like $\left\{z_{1}, \ldots, z_{s}, x_{1}, \ldots, x_{n}, y_{1}, \ldots, y_{m}\right\}$, it follows easily from the Standard Basis Theorem for Schur complexes and [2, Corollary V.1.14; 2 , Theorem II.4.11] that there is an embedding of complexes $i: \mathbf{L}_{\lambda / \mu} \varphi \hookrightarrow\left(\mathbf{L}_{\lambda} \psi\right)_{\tilde{\mu}}$, where $\left(\mathbf{L}_{\lambda} \psi\right)_{\tilde{\mu}}$ stands for the subcomplex of $\mathbf{L}_{\lambda} \psi$ spanned by the tableaux of shape $\lambda$ which are row-standard $\bmod Y$ (cf. [2, Definition V.1.8]) and such that each $z_{i}$ exactly occurs $\tilde{\mu}_{i}$ times (here, $\tilde{\mu}$ is the conjugate partition of $\mu$ ).

Remark 1.1. The embedding $i$ is not $\mathrm{GL}(H)$-equivariant but preserves the $\mathrm{GL}(G) \times \mathrm{GL}(F)$ structure we care for.

We now explain the reason for introducing the free $R$-module $H$.

Let $b^{(k)}=z_{1} \wedge z_{2} \wedge \cdots \wedge z_{k}, r=l(\mu)$, and $q=l(\lambda)$. Consider all sets $\left\{t_{r 1}, \ldots, t_{11}, t_{r 2}, \ldots, t_{12}, \ldots, t_{r q}, \ldots, t_{1 q}\right\}$, where the elements are nonnegative integers such that

(a) for every $i=1, \ldots, r, \sum_{j=1}^{q} t_{i j}=\mu_{i}$,

(b) if for every $j=1, \ldots, q, \nu_{j}$ stands for $\lambda_{j}-\sum_{i=1}^{r} t_{i j}$, then $\left(\nu_{1}, \ldots, \nu_{q}\right)$ is a partition $\nu$

(obviously, $\nu \subseteq \lambda$, and $|\nu|=|\lambda|-|\mu|$ ).

Definition 1.2. For every partition $\nu \subseteq \lambda$ such that $|\nu|=|\lambda|-|\mu|$, let $B(\lambda / \nu)$ denote the (finite) set consisting of the elements of $\Lambda_{\lambda / \nu} H$ of type

$$
\sum_{\beta_{r}, \ldots, \beta_{1}} b_{\beta_{r} t_{r 1}}^{\left(\mu_{r}\right)} \wedge \cdots \wedge b_{\beta_{1} t_{11}}^{\left(\mu_{1}\right)} \otimes \cdots \otimes b_{\beta_{r} t_{r i}}^{\left(\mu_{r}\right)} \wedge \cdots \wedge b_{\beta_{1} t_{1 i}}^{\left(\mu_{1}\right)} \otimes \cdots \otimes b_{\beta_{r} t_{r q}}^{\left(\mu_{r}\right)} \wedge \cdots \wedge b_{\beta_{1} t_{1 q}}^{\left(\mu_{1}\right)}
$$

for all possible choices of sets $\left\{t_{r 1}, \ldots, t_{11}, t_{r 2}, \ldots, t_{12}, \ldots, t_{r q}, \ldots, t_{1 q}\right\}$.

Here, $\sum_{\beta_{i}} b_{\beta_{i} t_{i 1}}^{\left(\mu_{i}\right)} \otimes \cdots \otimes b_{\beta_{i} t_{i q}}^{\left(\mu_{i}\right)}=\Delta\left(b^{\left(\mu_{i}\right)}\right)$ for every $i=1, \ldots, r$, with $\Delta$ the diagonal map $\Lambda^{\mu_{i}} H \rightarrow \Lambda^{t_{i 1}} H \otimes \cdots \otimes \Lambda^{t_{i q}} H$.

Remark 1.3. The elements of $B(\lambda / \nu)$ are in fact contained in $\left(\Lambda_{\lambda / \nu} H\right)_{\tilde{\mu}}^{U^{+}(H)}$; that is, they are $U^{+}(H)$-invariant and each $z_{i}$ occurs in them exactly $\tilde{\mu}_{i}$ times. $\left(U^{+}(H) \cong U^{+}\left(\mu_{1} ; R\right)\right.$ is the group of upper unitriangular matrices of order $\mu_{1}$ with entries in $R$.)

Lemma 1.4. $\psi=\varphi \oplus \zeta$, where $\zeta$ is the zero map $0 \rightarrow H$.

Definition 1.5. For every $b \in B(\lambda / \nu)$, let $\varphi(\nu, b)$ denote the map $\mathbf{\Lambda}_{\nu} \varphi \rightarrow$ $\left(\mathbf{L}_{\lambda} \psi\right)_{\tilde{\mu}}$, which is obtained by first restricting to $\boldsymbol{\Lambda}_{\nu} \varphi \otimes\{b\}$ the (obvious) morphism $\boldsymbol{\Lambda}_{\nu} \varphi \otimes \boldsymbol{\Lambda}_{\lambda / \nu} \zeta \rightarrow \boldsymbol{\Lambda}_{\lambda} \psi$ of [2, Definition V.1.11] and then composing with $d_{\lambda} \psi$. 
(For the definitions of the complexes $\Lambda_{.-}$and the map $d_{\lambda} \psi$, cf. [2, p. 262].)

What happens if we apply to a generator of $\operatorname{Im}(\varphi(\nu, b))$ the straightening law going from $\left\{x_{1}, \ldots, x_{n}, y_{1}, \ldots, y_{m}, z_{1}, \ldots, z_{s}\right\}$ to $\left\{z_{1}, \ldots, z_{s}, x_{1}, \ldots, x_{n}\right.$, $\left.y_{1}, \ldots, y_{m}\right\}$ ? Certainly we still have a $U^{+}(H)$-invariant element of $\left(\mathbf{L}_{\lambda} \psi\right)_{\tilde{\mu}}$, but since the tableau

$$
\begin{array}{lllllll}
z_{1} & z_{2} & z_{3} & \cdots & \cdots & z_{\mu_{1}} \\
z_{1} & z_{2} & z_{3} & \cdots & \cdots & z_{\mu_{2}} \\
z_{1} & z_{2} & z_{3} & \cdots & z_{\mu_{3}} \\
\vdots & & \multicolumn{2}{|c}{} \\
\vdots & & &
\end{array}
$$

(of shape $\mu$ ) is the only $U^{+}(H)$-invariant standard tableau containing $\tilde{\mu}_{i}$ copies of $z_{i}$ for every $i=1, \ldots, s$, we in fact get an element of $\operatorname{Im}(i)$ (cf. [2, Theorem in the appendix]).

Therefore, $\varphi(\nu, b)$ can be thought of as a map from $\mathbf{\Lambda}_{\nu} \varphi$ to $\mathbf{L}_{\lambda / \mu} \varphi$. This map will be the main ingredient in our construction.

\section{THE FILTRATION}

Definition 2.1. $\mathbf{M}_{\nu} \varphi$ is the subcomplex of the complex $\mathrm{L}_{\lambda / \mu} \varphi$ given by the sum $\sum_{\tau \geq \nu, b \in B(\lambda / \tau)} \operatorname{Im}(\varphi(\tau, b))$.

The subcomplexes $\mathbf{M}_{\nu} \varphi$, ordered lexicographically $\left(\nu_{1} \leq \nu_{2} \Rightarrow \mathbf{M}_{\nu_{2}} \varphi \subseteq\right.$ $\left.\mathbf{M}_{\nu_{1}} \varphi\right)$ will give the filtration of $\mathbf{L}_{\lambda / \mu} \varphi$ for which we are looking.

We denote by $\dot{\mathbf{M}}_{\nu} \varphi$ the subcomplex immediately preceding $\mathbf{M}_{\nu} \varphi$, i.e., $\dot{\mathbf{M}}_{\nu} \varphi$ $=\sum_{\tau>\nu, b \in B(\lambda / \tau)} \operatorname{Im}(\varphi(\tau, b))$.

Proposition 2.2. (i) $\left\{\mathbf{M}_{\nu} \varphi\right\}$ is an exhaustive filtration.

(ii) Each $\varphi(\nu, b): \mathbf{\Lambda}_{\nu} \varphi \rightarrow \mathbf{L}_{\lambda / \mu} \varphi$ induces a map $\mathbf{L}_{\nu} \varphi \rightarrow \mathbf{L}_{\lambda / \mu} \varphi / \dot{\mathbf{M}}_{\nu} \varphi$, indeed a map $\mathbf{L}_{\nu} \varphi \rightarrow \mathbf{M}_{\nu} \varphi / \dot{\mathbf{M}}_{\mu} \varphi$, denoted by $\bar{\varphi}(\nu, b)$.

Proof. (i) One can mimic the proof of [4, Proposition 3.1], provided one replaces each $\Lambda^{i}(F \oplus G)$ by $\Lambda^{i}(\varphi \oplus \zeta)$ and keeps in mind the peculiar nature of $\zeta$, as defined in Lemma 1.4 .

(ii) One can mimic the proof of [4, Lemma 3.6], reading $\Lambda^{i}(\varphi \oplus \zeta)$ instead of $\Lambda^{i}(F \oplus G)$.

We want to show that each $\mathbf{M}_{\nu} \varphi / \dot{\mathbf{M}}_{\nu} \varphi$ is isomorphic to the sum of exactly $\gamma(\lambda / \mu ; \nu)$ copies of $\mathbf{L}_{\nu} \varphi$. The idea is to reduce the case over $\mathbf{Z}$ (whence the case over every $R$ ) to the case over $Q$, where the result is known to be true (cf., e.g., $[10, \S 0.3 ; 9])$.

Combinatorially, $\gamma(\lambda / \mu ; \nu)$ is the number of standard tableaux of shape $\lambda / \nu$, filled with $\tilde{\mu}_{1}$ copies of $1, \tilde{\mu}_{2}$ copies of $2, \tilde{\mu}_{3}$ copies of 3 , etc., such that the associated word (formed by listing all entries from bottom to top in each column, starting from the leftmost column) is a lattice permutation (cf. $[5, \S 1]$ ).

To the above standard tableaux of shape $\lambda / \nu$, one can associate in a oneto-one way $\gamma(\lambda / \mu ; \nu)$ elements of $B(\lambda / \nu)$, thus obtaining a subset $B^{\prime}(\lambda / \nu)$ of $B(\lambda / \nu)$ such that $\left|B^{\prime}(\lambda / \nu)\right|=\gamma(\lambda / \mu ; \nu)$ (cf. [5, §3]).

Now notice that if in the first part of the proof of [4, Theorem 4.5] one replaces the standard tableau $d_{\nu}(a) \in L_{\nu} F$ by any linear combination $\sum_{j} c_{j} d_{\nu}\left(a_{j}\right)$ of standard tableaux, the very same argument shows:

After ordering the elements of $B^{\prime}(\lambda / \nu)$ in the way described in [5, §3], for 
every $b^{\prime} \in B^{\prime}(\lambda / \nu)$ one has that

$$
\begin{aligned}
& \varphi\left(\nu, b^{\prime}\right)\left(\sum c_{j} a_{j}\right) \in \dot{\mathbf{M}}_{\nu}+\sum_{b^{\prime \prime}<b^{\prime}} \operatorname{Im}\left(\varphi\left(\nu, b^{\prime \prime}\right)\right) \\
& \text { implies } \sum_{j} c_{j} d_{\nu}\left(a_{j}\right)=0, \quad \text { whence } c_{j}=0 \text { for every } j .
\end{aligned}
$$

(The proof of [4, Theorem 4.5] is given in the case $\mu$ is a rectangle but does not depend on the assumption $\mu_{1}=\cdots=\mu_{r}$, as already remarked in [5, §3].)

Once more, reading $\varphi \oplus \zeta$ instead of $F \oplus G$ in the above, (\$) becomes a statement like the following: After ordering the elements of $B^{\prime}(\lambda / \nu)$ in the way described in $[5, \S 3]$, for every $b^{\prime} \in B^{\prime}(\lambda / \nu)$,

$$
\bar{\varphi}\left(\nu, b^{\prime}\right): \mathbf{L}_{\nu} \varphi \rightarrow \mathbf{M}_{\nu} \varphi /\left(\dot{\mathbf{M}}_{\nu} \varphi+\sum_{b^{\prime \prime}<b^{\prime}} \operatorname{Im}\left(\varphi\left(\nu, b^{\prime \prime}\right)\right)\right)
$$

is injective over $\mathbf{Z}$.

Thus, over the field $\mathbf{Q}$, one is forced to conclude from the known decomposition $\sum_{\nu} \gamma(\lambda / \mu ; \nu) \mathbf{L}_{\nu} \varphi$ of $\mathbf{L}_{\lambda / \mu} \varphi$ that, for every $b \in B(\lambda / \nu)-B^{\prime}(\lambda / \nu)$,

$$
\operatorname{Im}(\varphi(\nu, b)) \subseteq \dot{\mathbf{M}}_{\nu} \varphi+\sum_{b^{\prime} \in B^{\prime}(\lambda / \nu)} \operatorname{Im}\left(\varphi\left(\nu, b^{\prime}\right)\right) .
$$

Mimicking the second part of the proof of [4, Theorem 4.5] (again ignoring the assumption $\mu_{1}=\cdots=\mu_{r}$ and reading $\varphi \oplus \zeta$ instead of $\left.F \oplus G\right)$, it follows that (\&) in fact holds over $\mathbf{Z}$.

Summarizing, we have proven that we can safely discard all maps $\varphi(\nu, b)$ with $b \in B(\lambda / \nu)-B^{\prime}(\lambda / \nu)$, for they do not add anything to the images of the $\varphi\left(\nu, b^{\prime}\right)$ with $b^{\prime} \in B^{\prime}(\lambda / \nu)$.

This completes the proof of the following theorem.

Theorem 2.3. $\mathbf{L}_{\lambda / \mu} \varphi$ has a universal filtration whose associated graded object is $\sum_{\nu} \gamma(\lambda / \mu ; \nu) \mathbf{L}_{\nu} \varphi$.

Remark 2.4. Further observations can be made on $B(\lambda / \nu), B^{\prime}(\lambda / \nu)$, and their elements, as well as on the quotients $\mathbf{M}_{\nu} \varphi / \dot{\mathbf{M}}_{\nu} \varphi$, in a way similar to that of $[5, \S 3]$.

\section{REFERENCES}

1. K. Akin, D. A. Buchsbaum, and J. Weyman, Resolutions of determinantal ideals: The submaximal minors, Adv. in Math. 39 (1981), 1-30.

2. __ Schur functors and Schur complexes, Adv. in Math. 44 (1982), 207-278.

3. M. Barnabei and A. Brini, The Littlewood-Richardson rule for co-Schur modules, Adv. in Math. 67 (1988), 143-173.

4. G. Boff, The universal form of the Littlewood-Richardson rule, Adv. in Math. 68 (1988), 40-63.

5. _ Characteristic-free decomposition of skew Schur functors, J. Algebra 125 (1989), 288297.

6. _ـ A remark on a paper by Barnabei and Brini, J. Algebra 139 (1991), 458-467.

7. T. Jozefiak, P. Pragacz, and J. Weyman, Resolutions of determinantal varieties and tensor complexes associated with symmetric and antisymmetric matrices, Astérisque 87-88 (1981), 109-189. 
8. H. J. Ko, The decomposition of Schur complexes, Trans. Amer. Math. Soc. 324 (1991), 255-270.

9. H. A. Nielsen, Tensor functors of complexes, Aarhus Univ. Preprint Series, no. 15, 1978.

10. P. Pragacz and J. Weyman, Complexes associated with trace and evaluation. Another approach to Lascoux's resolution, Adv. in Math. 57 (1985), 163-207.

11. M. Artale and G. Boffi, On a subcomplex of the Schur complex, preprint, 1991.

ITALIA

Dipartimento di Matematica, II Università di Roma, Via O. Raimondo, 00173 Roma, 